\title{
Is the Voter Model a Model for Voters?
}

\author{
Juan Fernández-Gracia, ${ }^{1, *}$ Krzysztof Suchecki, ${ }^{2}$ José J. Ramasco, ${ }^{1}$ Maxi San Miguel, ${ }^{1}$ and Víctor M. Eguíluz ${ }^{1}$ \\ ${ }^{1}$ IFISC (CSIC-UIB), 07122 Palma de Mallorca, Spain \\ ${ }^{2}$ Faculty of Physics and Center of Excellence for Complex Systems Research, Warsaw University of Technology, \\ Koszykowa 75, 00-662 Warsaw, Poland
}

(Received 4 September 2013; revised manuscript received 4 February 2014; published 18 April 2014)

\begin{abstract}
The voter model has been studied extensively as a paradigmatic opinion dynamics model. However, its ability to model real opinion dynamics has not been addressed. We introduce a noisy voter model (accounting for social influence) with recurrent mobility of agents (as a proxy for social context), where the spatial and population diversity are taken as inputs to the model. We show that the dynamics can be described as a noisy diffusive process that contains the proper anisotropic coupling topology given by population and mobility heterogeneity. The model captures statistical features of U.S. presidential elections as the stationary vote-share fluctuations across counties and the long-range spatial correlations that decay logarithmically with the distance. Furthermore, it recovers the behavior of these properties when the geographical space is coarse grained at different scales-from the county level through congressional districts, and up to states. Finally, we analyze the role of the mobility range and the randomness in decision making, which are consistent with the empirical observations.
\end{abstract}

Opinion dynamics focuses on the way different options compete in a population, giving rise to either consensus (every individual holding the same opinion or option) or coexistence of several opinions. Many theoretical efforts have been devoted to clarifying the implications on the macroscopic outcome, among other aspects, of different interaction mechanisms, different topologies of the interaction networks, the inclusion of opinion leaders or of zealots, and external fields [1,2]. To advance our understanding of social phenomena, these theoretical efforts need to be complemented by empirical [3-5] and experimental results [6-9]. In this context, elections offer an opportunity for contrasting models of opinion dynamics with empirical results [10]. On one hand, the data are publicly available in many countries, with a good level of spatial resolution and several temporal observations. On the other hand, there is evidence that voting behavior is strongly influenced by the social context of the individuals $[6,7,9,11-20]$. Thus it is natural to model electoral processes as systems of interacting agents with the aim of explaining the statistical regularities [21-38], as, for example, the universal scaling of the distribution of votes in proportional elections [21,22] or signatures of irregularities in the democratic process $[23,26]$.

In this Letter we assess the capacity of the voter model to capture real voter choices and propose a microscopic foundation for modeling voting behavior in elections. The model is based on social influence and recurrent mobility (SIRM): social influence will be modeled as a noisy voter model, while recurrent mobility serves as a proxy of the social context. In the voter model each agent updates its state by randomly copying the opinion of one of its neighbors [39-41]. We will consider that agents interact at home and at work locations according to their commuting pattern $[42,43]$. We first obtain the statistical features of the U.S. presidential elections and then we introduce and analyze the model.

Statistical regularities in elections.-The analysis focuses on U.S. presidential elections from 1980 to 2012 (see the Supplemental Material [44]) due to the combination of data availability and an almost bipartisan system. The vote share per county $v$ for either of the two main parties, that is, the percentage of votes in a county, is distributed following approximately a Gaussian distribution [Fig. 1(a)], consistent with observations in other countries [23]. The average vote share over all counties changes from election to election, but the width remains approximately constant for each year, with a standard deviation of $\sigma_{e} \simeq 0.11$. We also find that the spatial correlation of the vote shares decays logarithmically with the geographical distance [Fig. 1(b)], as reported previously for turnout and winner
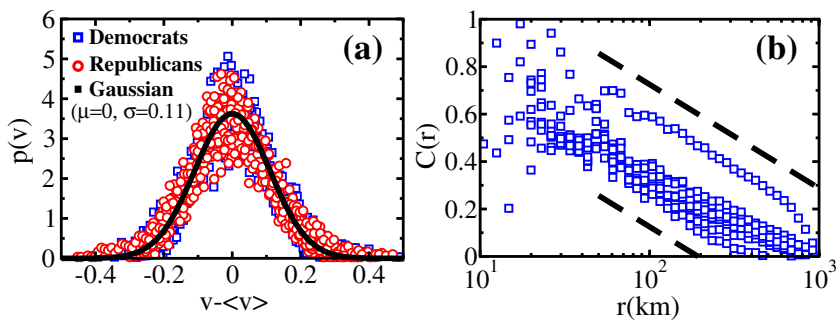

FIG. 1 (color online). U.S. electoral results 1980-2012. (a) County vote-share probability density functions. (b) Spatial vote-share correlations as a function of distance. The dashed lines indicate logarithmic decay. 
party vote shares $[24,25]$. The spatial correlation function is computed as

$$
C(r)=\left(\left.\left\langle v_{i} v_{j}\right\rangle\right|_{d\left(\vec{r}_{i}, \vec{r}_{j}\right)=r}-\langle v\rangle^{2}\right) /\left[\sigma^{2}(v)\right],
$$

where $\langle v\rangle$ is the average vote share over all the cells, $\sigma^{2}(v)$ its standard deviation, and $\left\langle v_{i} v_{j}\right\rangle_{d\left(\vec{r}_{j}, \vec{r}_{i}\right)=r}$ is averaged over pairs of cells separated a distance $r$. The stationarity of the vote-share dispersion and the logarithmic decay of the spatial correlations will be considered as generic of the fluctuations in electoral dynamics.

The model.-In the SIRM model $N$ agents live in a spatial system divided in nonoverlapping cells [45]. The agents are distributed among the different cells according to their residence cell. The number of residents in a particular cell $i$ is $N_{i}$. While many of these individuals may work at $i$, some others will work at different cells. This defines the fluxes $N_{i j}$ of residents of $i$ recurrently moving to $j$ for work. By consistency, $N_{i}=\sum_{j} N_{i j}$. The working population at cell $i$ is $N_{i}^{\prime}=\sum_{j} N_{j i}$ and the total population in the country is $N=\sum_{i j} N_{i j}$.

We describe the opinion of the agents by a binary variable $(+1$ or -1$)$. The number of individuals holding opinion +1 , living in county $i$ and working at $j$ is $V_{i j}$; thus, $V_{i}=\sum_{l} V_{i l}$ is the number of voters living in $i$ holding opinion $+1, V_{j}^{\prime}=\sum_{l} V_{l j}$ is the number of voters working at $j$ holding opinion +1 . We assume that each individual interacts with people living in her own location with a probability $\alpha$, while with probability $1-\alpha$ she interacts with individuals of her work place. Once an individual interacts with others, its opinion is updated following a noisy voter model $[15,39,40,46,47]$ : an interaction partner is chosen and the original agent copies her opinion imperfectly (with a certain probability of making mistakes). The evolution of the system can be expressed in terms of the transition rates

$$
\begin{aligned}
r_{i j}^{-}(\mathscr{V}) & =V_{i j}\left[\alpha \frac{N_{i}-V_{i}}{N_{i}}+(1-\alpha) \frac{N_{j}^{\prime}-V_{j}^{\prime}}{N_{j}^{\prime}}\right] \\
& +N_{i j} \frac{D}{2} \eta_{i j}^{-}(t), \\
r_{i j}^{+}(\mathscr{V})= & \left(N_{i j}-V_{i j}\right)\left[\alpha \frac{V_{i}}{N_{i}}+(1-\alpha) \frac{V_{j}^{\prime}}{N_{j}^{\prime}}\right]+N_{i j} \frac{D}{2} \eta_{i j}^{+}(t),
\end{aligned}
$$

where $\mathscr{V}=\left\{V_{i j}\right\}$ is the configuration of the system according to the set of variables $V_{i j}$, and $r_{i j}^{ \pm}(\mathscr{V})$ are the rates of change of $V_{i j}$ by one unit to $V_{i j} \pm 1$. These rates include recurrent mobility, and so they are different from those obtained for random diffusion processes [48]. The variables $\eta_{i j}^{ \pm}(t)$ are noise terms accounting for imperfect imitation, modeled as Gaussian noise with zero mean and $\left\langle\eta_{i j}^{a}(t) \eta_{k l}^{b}\left(t^{\prime}\right)\right\rangle=\delta\left(t-t^{\prime}\right) \delta_{a b} \delta_{i k} \delta_{j l}$ [49]. At the leading order, which corresponds to taking into account only the external noise coming from imperfect imitation, while the internal noise coming from the finite number of voters is neglected, the set of stochastic differential equations for $v_{i j}=V_{i j} / N_{i j}$ is

$$
\frac{d v_{i j}}{d t}=\alpha \sum_{l} A_{i j l} v_{i l}+(1-\alpha) \sum_{l} B_{i j l} v_{l j}+D \eta_{i j}(t),
$$

with $A_{i j l}=\left(N_{i l}\right) /\left(N_{i}\right)-\delta_{j l}$ and $B_{i j l}=\left(N_{l j}\right) /\left(N_{j}^{\prime}\right)-\delta_{l i}$ (see the Supplemental Material [44]). The first term on the right hand side describes interactions among agents who live in $i$ and work elsewhere, while the second term follows from the interactions among agents who work in $j$ and live elsewhere. The last term is noise coming from a combination of $\eta_{i j}^{+}(t)$ and $\eta_{i j}^{-}(t): \eta_{i j}(t)$ is also a Gaussian noise with zero mean and $\left\langle\eta_{i j}(t) \eta_{k l}\left(t^{\prime}\right)\right\rangle=\delta\left(t-t^{\prime}\right) \delta_{i k} \delta_{j l}$. This term represents imperfect imitation and accounts for the combined effect of all other influences different from the interaction between peers. This includes opinion drift, local media, or free will of the individuals. When $D \neq 0$, the microscopic rules lead to a noisy diffusive equation, in agreement with previous models of mesoscopic electoral dynamics [24,25]. The equation corresponds to an EdwardsWilkinson equation on a disordered medium, described by the coupling matrices $A$ and $B$. In the absence of imperfect imitation $(D=0)$, Eq. (3) can be written as a Laplacian $(d / d t) \vec{v}=\mathscr{L} \vec{v}$. This implies a homogeneous asymptotic configuration and the existence of a globally conserved variable, namely, the total number of voters holding opinion $+1, V=\sum_{i j} V_{i j}[44,50]$.

When simulating the model, we integrate the stochastic process by updating the values of the number of agents holding opinion +1 in each cell $i j, V_{i j}$, using binomial distributions with the rates in Eq. (2). At each Monte Carlo step we update all cells in a random order. Therefore we simulate the original master equation of the process.

Model calibration.-We apply the model to the U.S. presidential elections, identifying the cells with the counties. The populations and commuting fluxes $N_{i j}$ are obtained from the 2000 Census [51] and are input data for the SIRM model. This framework can be applied to any country or territorial division (counties, municipalities, provinces, states, etc.). Besides these data, there are two free parameters: $D$ and $\alpha$. The parameter $\alpha$ provides a measure of the relative intensity and duration of social relations at work and at home. According to the survey on time use from the Bureau of Labor Statistics [52], the average individual spends almost eight hours daily at work, and the rest of time at her home location. Out of this home time, close to another eight hours are spent sleeping. Thus $\alpha$ will be set at $1 / 2$, although other values give rise to qualitatively similar results (Fig. S10 in the Supplemental Material [44]) as long as $\alpha \neq 0$ or 1 , in which cases the system would consist of disconnected patches and thus there would not be any spatial diffusion.

To calibrate the noise intensity $D$, the SIRM model is run for a set of values of $D$, taking as initial condition the results for the elections of the year 2000. The system is 
evolved for $1000 \mathrm{MC}$ steps, and then the standard deviation $\sigma$ of the vote-share distribution is measured [panel (a) in Fig. 2]. Best agreement is obtained for $D=0.03$, which is taken as the level of noise for the simulations of the model. When the noise intensity is too low, we find basically a diffusive process, where the voteshare distribution narrows and the correlations grow $(D=0.005$ in Fig. 2). In contrast, for larger $D$ the noise is dominating the results ( $D=0.35$ in Fig. 2$)$. The voteshare distribution widens as time goes by and the spatial correlations vanish. For $D=0.03$ the standard deviation of the vote-share distribution of the model has the same value as the data. Not only is the standard deviation matched, but also the shape of the vote-share distribution agrees with the empirical one. The distribution, in addition, becomes stationary in time. Furthermore, although we did not take spatial correlations into account for the calibration, they show a stationary logarithmic decay for this value of noise intensity $D$.
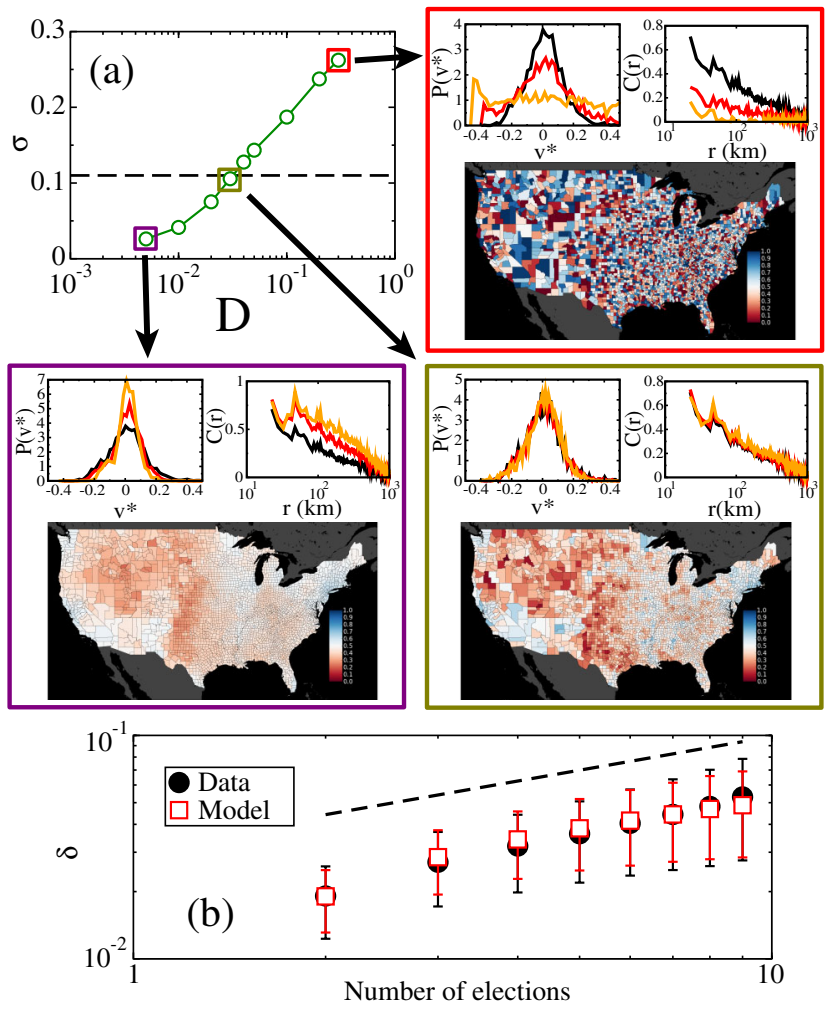

FIG. 2 (color online). (a) Vote-share standard deviation versus noise intensity $D$. The dashed black line marks the dispersion of the empirical data $\left(\sigma_{e}=0.11\right)$. Boxes surrounding the main plot display results obtained with the level of noise marked as squares and include the distribution of vote shares shifted to have zero mean, along with their spatial correlations. Darker (black) curves are initial conditions. Results are shown for increasing time steps from darker to lighter: 10 and $20 \mathrm{MC}$ steps (top right panel); 100 and $200 \mathrm{MC}$ steps (bottom right panel); 40 and $140 \mathrm{MC}$ steps (bottom left panel). (b) The average dispersion in the Democrat vote share is plotted versus the number of elections. Best agreement is obtained for $2.5 \mathrm{MC}$ steps/year.
Finally, we set the equivalence between the Monte Carlo steps and the real time between elections [Fig. 2(b)]. Equation (3) is written in arbitrary time units and is related to the updates by $d t=1 / N$ [53]. Sets of electoral results are produced with the model, with $D=0.03$ and with a fixed number of Monte Carlo steps between elections. Then the standard deviation $\delta$ of the vote-share trajectory for each county, as a function of the number of consecutive elections, is computed. Averaging over all different counties and comparing with empirical data, we find that both curves grow as $\sqrt{n}$, where $n$ is the number of elections considered (error bars correspond to the dispersion of $\delta$ across counties), reminiscent of a random walk. Both curves have the best overlap when we set $10 \mathrm{MC}$ steps/election (equivalently, 2.5 MC steps/year).

Results.-The stochasticity of the model introduces uncertainty in the temporal evolution of the vote shares, as can be
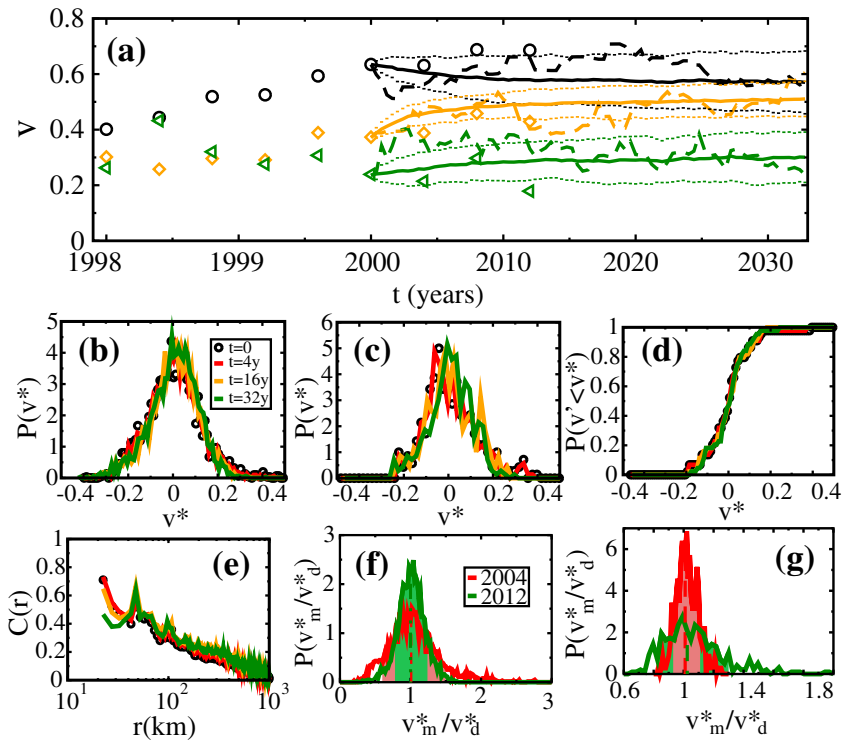

FIG. 3 (color online). Parameters of the simulation are $\alpha=1 / 2$, $D=0.03$. (a) Time traces of the vote shares for Democrats in different counties: one with high population, Los Angeles, California [darkest (black) symbols and curves, $9.5 \times 10^{6}$ inhabitants]; one with a medium population, Blane, Idaho [light gray (orange), $19 \times 10^{3}$ inhabitants]; and one with low population, Loving, Texas [medium gray (green) line, 67 inhabitants]. Symbols represent data and dashed lines represent the results of a single realization of the model with initial conditions taken from year 2000 data; solid lines represent the average of 100 realizations of the model and dotted lines their standard deviation. (b), (c), and (d) Democratic vote-share probability density functions, showing the cumulative probability density functions (PDF) [except for (d) that shows the cumulative PDF] as predicted by the model for counties, congressional districts, and states, respectively. Initial condition at $t=0$ (black circles): vote shares obtained from the 2000 elections. (e) Vote-share spatial correlations as a function of the distance. (f) and (g) Distribution of ratio between model predictions and data observations for the Democrat vote shares at county level (f) and for congressional districts (g). The colored areas mark $80 \%$ confidence intervals. 


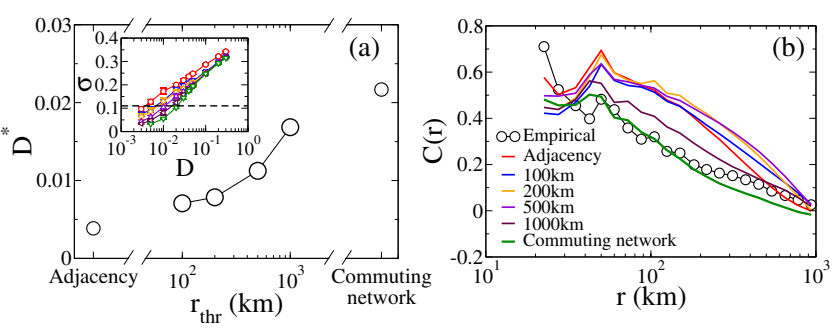

FIG. 4 (color online). (a) Calibrated noise intensity $D^{*}$ on networks thresholded at different distances. (b) Vote-share spatial correlations on different networks.

appreciated for three counties in Fig. 3(a). Once the average value is discounted, the shape of the distribution of vote shares is similar to the one observed in the empirical data [Fig. 3(b)]: the stationarity and the particular functional shape of the distributions are captured by the model. This occurs not only at county level [Fig. 3(b)], but also at other coarse-grained geographical scales such as congressional districts [(Fig. 3(c)] and states [Fig. 3(d)]. This relates to the ability of the model to properly capture the spatial correlations in the data [see Fig. 3(e) and Fig. S7 in the Supplemental Material [44] for a comparison with reshuffled data].

The goodness of the model is also assessed by a direct comparison between model predictions and data for voteshare fluctuations. In Figs. 3(f) and 3(g), we show the distribution of the ratios between model and data of the vote-share deviations from the national average, $v_{i}-\langle v\rangle$, where $\langle\cdot\rangle$ denotes spatial average (not average over realizations of the model). We evolve the model for an election, starting with the initial conditions from the electoral results from year 2000, and compare with the electoral results from year 2004, finding that $80 \%$ of the ratios fall between 0.6 and 1.5. These numbers become 0.9 and 1.1 at the congressional district level, attesting the quality of the model predictions.

As a final point, we investigate the role played by the mobility network on the model results. The links connecting only geographically neighboring counties can be extracted and used as a baseline network. The rest of the links are then added, filtering by the distance that separates the centroid of the residence county to that of the work county. The result of performing this operation is a network that includes more and more links as the threshold of the filter is increased. The model has to be calibrated for each new network [Fig. 4(a)]. Once the optimal value for the noise level of the imperfect imitation $D^{*}$ is found, the model simulations running on different networks can be compared with the empirical data. In Fig. 4(b), we show how the vote-share spatial correlations change when the network is modified. Long links are important to recover correlation values similar to those observed empirically.

Discussion.-We have introduced a microscopic model for opinion dynamics, the main ingredients of which are social influence (modeled as a noisy voter model), mobility, and population heterogeneity. The model can be approximated by a noisy diffusion equation on an anisotropic substrate that is given by the highly heterogeneous population and commuting data. It reproduces generic features of the vote-share fluctuations observed in data coming from three decades of presidential elections. It is important to note that the model is not aimed at predicting the winning party, only the local fluctuations over the national average vote share. In this sense, it is able to capture the empirical distributions of vote-share fluctuations, the spatial correlations, and even the evolution of the local vote-share fluctuations. This agreement between model predictions and empirical data is maintained when the geographical areas considered are coarse grained, showing thus that the model accounts for the main mechanisms at play in the dynamics of the system at different scales. We have studied, as well, the relevance of the mobility range for the quantitative agreement of the model. Despite the various heterogeneity sources of the system (population, geography, topology, and commuter fluxes), the model still displays logarithmic spatial correlations as in a two-dimensional diffusion [54]. This robustness is connected to the spatial decay of the cells $[44,55]$. The field of random walks on heterogeneous media could also provide valuable insight [56].

The present Letter offers-with the use of demographic data as input-a comparison of a theoretical model with real data, which is used both for calibration and to evaluate the results. It proposes a path for further research in opinion dynamics, as it establishes a method to bridge the gap existing between microscopic mechanisms of social interchange and macroscopic results of surveys and electoral processes. One limitation of the work is the use of census data, which translates in a lack of fine structure for the interaction network. The use of digital data will provide the necessary information to fill this gap. Another important issue is the dynamics of the average vote share. To this end further elements need to be included, as, for example, the effects of social and mass media.

J. F.-G. receives economic support from the Conselleria d'Educació, Cultura i Universitats of the Government of the Balearic Islands and the ESF. J. J. R. acknowledges funding from the Ramón y Cajal program of the Spanish Ministry of Economy (MINECO). Partial support was received from MINECO and FEDER through projects FIS2011-24785 and FIS2012-30634 and from the EU Commission through the FP7 projects EUNOIA and LASAGNE.

* Corresponding author. juanf@ifisc.uib-csic.es

[1] C. Castellano, S. Fortunato, and V. Loreto, Rev. Mod. Phys. 81, 591 (2009).

[2] M. San Miguel, V. M. Eguíluz, R. Toral, and K. Klemm, Comput. Sci. Eng. 7, 67 (2005).

[3] L. Lizana, N. Mitarai, K. Sneppen, and H. Nakanishi, Phys. Rev. E 83, 066116 (2011).

[4] A. Kandler, R. Unger, and J. Steele, Phil. Trans. R. Soc. B 365, 3855 (2010). 
[5] D. M. Abrams and S. H. Strogatz, Nature (London) 424, 900 (2003).

[6] R. M. Bond, C. J. Fariss, J. J. Jones, A. D. I. Kramer, C. Marlow, J. E. Settle, and J. H. Fowler, Nature (London) 489, 295 (2012).

[7] D. Centola, Science 329, 1194 (2010).

[8] A. C. Gallup, J. J. Hale, D. J. T. Sumpter, S. Garnier, A. Kacelnik, J. R. Krebs, and I. D. Couzin, Proc. Natl. Acad. Sci. U.S.A. 109, 7245 (2012).

[9] J. Lorenz, H. Rauhut, F. Schweitzer, and D. Helbing, Proc. Natl. Acad. Sci. U.S.A. 108, 9020 (2011).

[10] S. Fortunato and C. Castellano, Phys. Today 65, No. 10, 74 (2012).

[11] B. C. Straits, Publ. Opin. Q. 54, 64 (1990).

[12] C. B. Kenny, Am. J. Pol. Sci. 36, 259 (1992).

[13] P. A. Beck, R. J. Dalton, S. Greene, and R. Huckfeldt, Am. Pol. Sci. Rev. 96, 57 (2002).

[14] A. S. Zuckerman, The Social Logic Of Politics: Personal Networks As Contexts For Political Behavior (Temple University Press, Philadelphia, 2005).

[15] J.H. Fowler, in Social Logic of Politics, edited by A. Zuckerman (Temple University Press, Philadelphia, 2005), pp. 269-287.

[16] P. F. Lazarsfeld, B. Berelson, and H. Gaudet, The People's Choice: How the Voter Makes Up His Mind in a Presidential Campaign (Columbia University Press, New York, 1948).

[17] E. M. Rogers, in Diffusion of Innovations (Free Press, New York, 1995).

[18] N. A. Christakis and J. H. Fowler, N. Engl. J. Med. 357, 370 (2007).

[19] A. L. Hill, D. G. Rand, M. A. Nowak, and N. A. Christakis, Proc. Biol. Sci. 277, 3827 (2010).

[20] L. Rendell, R. Boyd, D. Cownden, M. Enquist, K. Eriksson, M. W. Feldman, L. Fogarty, S. Ghirlanda, T. Lillicrap, and K. N. Laland, Science 328, 208 (2010).

[21] S. Fortunato and C. Castellano, Phys. Rev. Lett. 99, 138701 (2007).

[22] A. Chatterjee, M. Mitrović, and S. Fortunato, Sci. Rep. 3, 1049 (2013).

[23] P. Klimek, Y. Yegorov, R. Hanel, and S. Thurner, Proc. Natl. Acad. Sci. U.S.A. 109, 16469 (2012).

[24] C. Borghesi and J.-P. Bouchaud, Eur. Phys. J. B 75, 395 (2010).

[25] C. Borghesi, J.-C. Raynal, and J.-P. Bouchaud, PLoS One 7, e36289 (2012).

[26] R. Enikolopov, V. Korovkin, M. Petrova, K. Sonin, and A. Zakharov, Proc. Natl. Acad. Sci. U.S.A. 110, 448 (2013).

[27] R. N. Costa Filho, M. P. Almeida, J. S. Andrade, and J. E. Moreira, Phys. Rev. E 60, 1067 (1999).

[28] R. N. Costa Filho, M. P. Almeida, J.E. Moreira, and J. S. Andrade, Jr., Physica (Amsterdam) 322A, 698 (2003).

[29] L. E. Araripe and R. N. Costa Filho, Physica (Amsterdam) 388A, 4167 (2009).

[30] A. T. Bernardes, D. Stauffer, and J. Kertész, Eur. Phys. J. B 25, 123 (2002).

[31] M. L. Lyra, U. M. S. Costa, R. N. Costa-Filho, and J. S. Andrade, Europhys. Lett. 62, 131 (2003).

[32] G. Travieso and L. da Fontoura Costa, Phys. Rev. E 74, 036112 (2006).

[33] L. E. Araripe, R. N. Costa Filho, H. J. Herrmann, and J. S. Andrade, Int. J. Mod. Phys. C 17, 1809 (2006).
[34] M. C. Gonzalez, A. O. Sousa, and H. J. Herrmann, Int. J. Mod. Phys. C 15, 45 (2004).

[35] C. A. Andresen, H. F. Hansen, G. L. Vasconcelos, and J. S. Andrade, Int. J. Mod. Phys. A 19, 1647 (2008).

[36] H. Hernández-Saldaña, Physica (Amsterdam) 388A, 2699 (2009).

[37] N. A. Araújo, J. S. Andrade, and H. J. Herrmann, PLoS One 5, e12446 (2010).

[38] J. Kim, E. Elliott, and D.-M. Wang, Electoral Stud. 22, 741 (2003).

[39] R. Holley and T. M. Liggett, Ann. Probab. 3, 643 (1975).

[40] F. Vazquez and V. M. Eguíluz, New J. Phys. 10, 063011 (2008).

[41] K. Suchecki, V. M. Eguíluz, and M. San Miguel, Phys. Rev. E 72, 036132 (2005).

[42] M. C. González, C. Hidalgo, and A.-L. Barabási, Nature (London) 453, 779 (2008).

[43] C. Song, Z. Qu, N. Blumm, and A.-L. Barabási, Science 327, 1018 (2010).

[44] See Supplemental Material at http://link.aps.org/ supplemental/10.1103/PhysRevLett.112.158701, which includes Refs. [49, 51], for more details about the data sets and the derivation of the equations.

[45] This approach is based on a division of the space in metapopulations. L. Sattenspiel and K. Dietz, Math. Biosci. 128, 71 (1995); D. Balcan, V. Colizza, B. Gonçalves, H. Hu, J. J. Ramasco, and A. Vespignani, Proc. Natl. Acad. Sci. U.S.A. 106, 21484 (2009); M. Tizzoni, P. Bajardi, C. Poletto, J. J. Ramasco, D. Balcan, B. Gonçalves, N. Perra, V. Colizza, and A. Vespignani, BMC Med. 10, 165 (2012).

[46] V. Sood and S. Redner, Phys. Rev. Lett. 94, 178701 (2005).

[47] P. L. Krapivsky, S. Redner, and E. Ben-Naim, in A Kinetic View of Statistical Physics (Cambridge University Press, Cambridge, England, 2010).

[48] A. Baronchelli and R. Pastor-Satorras, J. Stat. Mech. (2009) L11001.

[49] M. A. Rodríguez, L. Pesquera, M. San Miguel, and J. M. Sancho, J. Stat. Phys. 40, 669 (1985).

[50] K. Klemm, M. A. Serrano, V. M. Eguíluz, and M. San Miguel, Sci. Rep. 2, 292 (2012).

[51] United States Census Bureau, http://www.census.gov.

[52] Bureau of Labor Stastistics, American Time Use Survey for 2012, http://www.bls.gov/tus.

[53] In the derivation of the master equation we set the differential of time to be $\delta t=1 / N$. This leads to Eq. (3), which is written in arbitrary units. The choice of $\delta t=1 / N$ makes the arbitrary time unit identifiable to the MC step. Thus in the case of the full commuting network, where $2.5 \mathrm{MC}$ steps correspond to a year, the arbitrary time unit in the Langevin equation corresponds to $0.4 \mathrm{yr}$. In order to have the Langevin equation written such that the time unit is $1 \mathrm{yr}$, one should rescale time by a factor $a=2.5$, and both the deterministic part of the equation and the noise term should be properly rescaled.

[54] S.-K. Ma, in Statistical Mechanics (World Scientific, Singapore, 1985).

[55] S. A. Marvel, T. Martin, C. R. Doering, D. Lusseau, and M. E. J. Newman, arXiv:1310.2636.

[56] D. Brockmann, L. Hufnagel, and T. Geisel, Nature (London) 439, 462 (2006). 